\title{
AN INVERSE METHOD WITH REGULARITY CONDITIÓN FOR TRANSONIC AIRFOIL DESIGN
}
Zhu Ziqiang, Xia Zhixun, and Wu Liyi Inst. of Fluid Mechanics
Beijing Univ. of Aeronautics and Astronautics
Beijing 100083, China

\section{ABSTRACT}

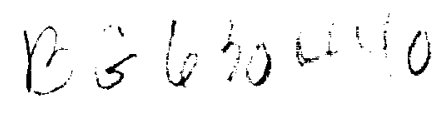

It is known from the Lighthill's exact solution of the incompressible inverse problem that in the inverse design problem the surface pressure distribution and the free stream speed can not both be prescribed independently. This implies the existence of a constraint (regularity condition) on the prescribed pressure distribution. The same constraint exists at compressible speeds. In this paper, a well-posed inverse design method for transonic airfoil is presented. In the method, the target pressure distribution contains a free parameter that is adjusted during the computation to satisfy the regularity condition derived in this paper. A few design results are presented here in order to demonstrate the capability of the method.

\section{INTRODUCTION}

Recently, a number of design methods have been developed and used for the design of transonic airfoils and wings. Slooff ${ }^{\prime}$ reviewed these methods and divided them into three major categories: indirect, inverse, and aerodynamic optimization. Indirect methods are characterized by the fact that the designer has no control over either the aerodynamic quantities or the geometry. The hodograph and fictitious gas methods are in this category. In inverse methods, the classical inverse problem of aerodynamics is solved. The designer specifies an arbitrary pressure distribution on an airfoil or wing, while the geometry of the airfoil or the wing that realizes the given pressure distribution is determined as the result of the solution. Aerodynamic optimization methods are those in which a nonlinear optimization algorithm is linked with a flow analysis code to minimize or maximize some aerodynamic object functions such as the lift-to-drag ratio.

The conventional inverse design methods are the most used in the industry application. The currently existing inverse methods for transonic airfoil design can be subdivided into two categories: (a) methods utilizing Dirichlet-type boundary conditions derived from the target pressure distribution; (b) methods utilizing Neumann-type boundary conditions in combination with some geometry correction procedure (residual- correction method).

In fact, in inverse problems, both a Dirichlet- and a Neumann-type boundary condition must be satisfied on the airfoil contour to be determined. This gives rise to a nonlinear problem with unknown boundary to be solved iteratively. In the first approach, the required target pressure distribution is imposed on an initial airfoil as a

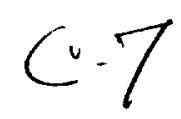


Dirichlet boundary condition, while airfoil geometry corrections are derived by integrating (either explicitly, or in some implicit manner) the transpiration mass flow over the initial airfoil. The Neumann boundary condition is satisfied at the end of the iterations.

In the second approach, the pressure distribution on an initial airfoil is determined by the use of an analysis code (Neumann boundary condition), and the residuals (i.e. differences between target pressure distribution and pressure distribution on the airfoil of the current iteration) are transformed to airfoil geometry corrections using some relatively simple approximate inverse methods. Here, the Dirichlet boundary condition is satisfied at the end of the iterations.

The main advantage of the iterative Dirichlet-type method is rapid convergence, provided closure and regularity conditions are satisfied. This kind of methods has been developed by Volpe and Melnik, ${ }^{2}$ Carlson ${ }^{3}$ and Tranen ${ }^{4}$ for airfoils, Henne ${ }^{5}$ for wing, and Shankar ${ }^{6}$ for wing / body designs. But in most of these methods, the regularity condition is obviously not taken into account.

The main advantage of the residual-correction method is its simplicity. Only a small investment is required, because any existing transonic analysis code can be used without modification. Efforts can be concentrated on coding a simple approximate inverse routine base on a suitable approach, and coupling this to the analysis code. Another advantage of this kind of methods is that the analysis code can be easily replaced with more advanced codes when they become available. This approach was used by Davis, ${ }^{7}$ McFadden, ${ }^{8}$ Fray et al, ${ }^{9}$ Greff and Mantel. ${ }^{10}$ Takanashi ${ }^{11}$ has developed a three-dimensional transonic wing design method based on this approach. The geometry correction problem is formulated in a three-dimensional transonic small-disturbance integral equation form and is numerically solved.

It was demonstrated by Lighthill ${ }^{12}$ that a unique and correct solution to the inverse problem of $2-D$, incompressible flow generally does not exist unless the prescribed speed distribution satisfies a certain integral constraint (regularity condition) arising from the requirement that the speed in the free stream be equal to one (or any other specified value). Thus, in order to assure that a solution to the inverse problem exists, some freedom must be permitted in the prescribed pressure distribution to allow the regularity condition to be satisfied. This can be accomplished by introducing a parameter into prescribed pressure distribution. In transonic flow the similar constraints have never been properly formulated. Volpe and Melnik ${ }^{2}$ proposed an inverse design method for transonic flow which was aimed at providing a treatment of such kind of constraints on the target speed distribution numerically.

In this paper, we formulate a well-posed inverse design method for transonic airfoil. This method is a residual-correction type approach, in which the procedure to determine the geometry correction is similar to the procedure used by Takanashi for his three-dimensional wing design method. An integral constraint (regularity condition) is derived and used in the method. Thus, a parameter is introduced into the target pressure distribution, and this parameter is determined as part of the solution according to the regularity condition. 


\section{FORMULATION OF THE INVERSE PROBLEM}

The full potential equation can be written in terms of the perturbation velocity potential as

$$
\left(1-M_{\infty}^{2}\right) \bar{\varphi}_{\overline{x x}}+\bar{\varphi}_{\bar{z} \bar{z}}=K \frac{\partial}{\partial \bar{x}}\left(\frac{1}{2} \bar{\varphi}_{\bar{x}}^{2}\right)+\bar{H}
$$

Where $\mathrm{M}_{\infty}$ is the free stream Mach number, $\mathrm{K}\left(\gamma, \mathrm{M}_{\infty}\right)$ is a transonic similarity parameter, $y$ is the ratio of specific heats, $(\bar{x}, \bar{z})$ is a Cartesian coordinate system, and $\bar{H}$ represents all the higher order terms.

the tangency condition on the airfoil may be written as

$$
\bar{\varphi}_{\bar{i}}(\bar{x}, \pm 0)=\overline{\mathrm{f}}_{ \pm}^{\prime}(\bar{x})+\bar{Q}
$$

where $\overline{\mathrm{f}}_{+}(\bar{x})$ and $\overline{\mathrm{f}}_{-}(\bar{x})$ are the equations of the upper and lower airfoil surfaces respectively, and $\bar{Q}$ represents all the higher order terms.

the pressure coefficients on the airfoil surface are expressed as

$$
C_{p \pm}(\bar{x})=-2 \bar{\varphi}_{\bar{x}}(\bar{x}, \pm 0)+\bar{S}
$$

where $\bar{S}$ represents all the higher order terms.

In a residual-correction method, the solution $\bar{\varphi}(\vec{x}, \vec{z})$ of Eq. (1) for an initial airfoil $\overline{\mathrm{f}}_{ \pm}(\bar{x})$ has been obtained by means of an existing analysis code, the objective here is to determine the amount of the geometry correction $\Delta \overline{\mathrm{f}}_{ \pm}(x)$ corresponding to the pressure difference $\Delta C_{p \pm}(x)$ between the specified and calculated pressure. If a small perturbation $\Delta \bar{\varphi}(\bar{x}, \bar{z})$ is further introduced, we can obtain the potential equations for $\Delta \bar{\varphi}(\bar{x}, \bar{z})$ according to the transonic small-disturbance theory

$$
\begin{gathered}
\Delta \varphi_{x x}+\Delta \varphi_{z z}=\frac{\partial}{\partial x}\left[\frac{1}{2}\left(\varphi_{x}+\Delta \varphi_{x}\right)^{2}-\frac{1}{2} \varphi_{x}^{2}\right] \\
\Delta \varphi_{z}(x, \pm 0)=\Delta \mathrm{f}_{ \pm}^{\prime}(x) \\
\Delta C_{p \pm}(x)=-2 \frac{\beta^{2}}{K} \Delta \varphi_{x}(x, \pm 0)
\end{gathered}
$$

here $\beta=\sqrt{1-M_{\infty}^{2}}$, and the new variables have been introduced as

$$
x=\bar{x}, \quad z=\beta \bar{z}, \quad \varphi(x, z)=\left(K / \beta^{2}\right) \bar{\varphi}(\bar{x}, \bar{z}), \quad \mathrm{f}_{ \pm}(x)=\left(K / \beta^{3}\right) \overline{\mathrm{f}}_{ \pm}(\bar{x})
$$

By applying Green's theory to Eq.(4) and introducing a decay function similar to that used by $\mathrm{N} \phi \mathrm{rstrud}^{13}$, we can get the integral equations

$$
\begin{aligned}
\Delta u_{s}(x)= & \frac{1}{\pi} \int_{0}^{1} \Psi_{x}(x, 0 ; \xi, 0) \Delta w_{s}(\xi) d \xi+G_{s}(x) \\
& -\frac{1}{\pi} \int_{0}^{1}\left[I_{s}(x, \xi,+0) G(\xi,+0)+I_{s}(x, \xi,-0) G(x,-0)\right] d \xi \\
\Delta w_{a}(x)= & \frac{1}{\pi} \int_{0}^{1} \frac{\Delta u_{a}(\xi)}{\xi-x} d \xi
\end{aligned}
$$




$$
-\frac{1}{\pi} \int_{0}^{1}\left[I_{a}(x, \xi,+0) G(\xi,+0)-I_{a}(x, \xi,-0) G(x,-0)\right] d \xi
$$

here

$$
\begin{gathered}
\Delta u_{s}(x)=\Delta \varphi_{x}(x,+0)+\Delta \varphi_{x}(x,-0) \\
\Delta u_{a}(x)=\Delta \varphi_{x}(x,+0)-\Delta \varphi_{x}(x,-0) \\
\Delta w_{s}(x)=\Delta \varphi_{z}(x,+0)-\Delta \varphi_{z}(x,-0) \\
\Delta w_{a}(x)=\Delta \varphi_{z}(x,+0)+\Delta \varphi_{z}(x,-0) \\
\Psi(x, z ; \xi, \zeta)=\ln \left[(x-\xi)^{2}+(z-\zeta)^{2}\right]^{\frac{1}{2}} \\
G(x, z)=\frac{1}{2}\left[\left(\varphi_{x}+\Delta \varphi_{x}\right)^{2}-\varphi_{x}^{2}\right] \\
I_{s}(x, \xi, \pm)=\int_{0}^{\infty} \Psi_{\zeta x}(x, 0 ; \xi, \zeta) \exp \left[-2 R_{ \pm}(\xi) \zeta\right] d \xi \\
I_{a}(x, \xi, \pm)=\int_{0}^{\infty} \Psi_{\zeta z}(x, 0 ; \xi, \zeta) \exp \left[-2 R_{ \pm}(\xi) \zeta\right] d \xi \\
R{ }_{ \pm}(x)=\left|\mathrm{f}_{ \pm}^{\prime \prime}(x) / \varphi_{x}(x, \pm 0)\right|
\end{gathered}
$$

For convenience, the correction function $\Delta \mathrm{f}_{ \pm}(\mathrm{x})$ is split into symmetric $\Delta \mathrm{f}_{\mathrm{s}}(\mathrm{x})$ and antisymmetric $\Delta \mathrm{f}_{\mathrm{a}}(\mathrm{x})$ parts

$$
\begin{aligned}
& \Delta \mathrm{f}_{s}(x)=\Delta \mathrm{f}_{+}(x)-\Delta \mathrm{f}_{-}(x) \\
& \Delta \mathrm{f}_{a}(x)=\Delta \mathrm{f}_{+}(x)+\Delta \mathrm{f}_{-}(x)
\end{aligned}
$$

Since $\Delta \mathrm{f}_{\mathrm{a}}^{\prime}(\mathrm{x})=\Delta \mathrm{w}_{\mathrm{a}}(\mathrm{x})$, the antisymmetric part can be determined by direct evaluation of the right-hand side of Eq. (9). On the other hand, since $\Delta \mathrm{f}_{\mathrm{s}}^{\prime}(\mathrm{x})=\Delta \mathrm{w}_{\mathrm{s}}(\mathrm{x})$. the symmetric part must be solved implicitly. Consequently, the correction $\Delta f_{ \pm}(x)$ is obtained by integrating $\Delta \mathrm{w}_{\mathrm{s}}(\mathrm{x})$ and $\Delta \mathrm{w}_{\mathrm{a}}(\mathrm{x})$ with respect to $\mathrm{x}$.

\section{CONSTRAINTS FOR INVERSE PROBLEM}

In the incompressible flow, it was demonstrated by Lighthill, ${ }^{12}$ using conformal mapping method, that the geometry of an airfoil for a given speed distribution can be determined only if the prescribed speed distribution satisfied the following three integral constraints

$$
\begin{gathered}
\int_{0}^{2 \pi} \log \left|\frac{q_{0}}{q_{\infty}}\right| d \omega=0 \\
\int_{0}^{2 \pi} \log \left|\frac{q_{0}}{q_{\infty}}\right| \cos \omega d \omega=0 \\
\int_{0}^{2 \pi} \log \left|\frac{q_{0}}{q_{\infty}}\right| \sin \omega d \omega=0
\end{gathered}
$$

here $q_{0}$ is the prescribed speed distribution on the airfoil surface, $q_{\infty}$ is the speed at infinity, $\omega$ is the polar angle in the transformed plane. The first constraint known as regularity condition is a consequence of the fact that the speed at infinity is $q_{\infty}$. Eqs. (21) and 
(22) together express that the airfoil is a closed contour and the angle of incidence is zero (or any other specified value).

In order to formulate the regularity condition for transonic flow, differentiating both sides of $\mathrm{Eq}(4)$ with respect to $\mathrm{x}$, we have

$$
\nabla^{2} V(x, z)=\frac{\partial^{2} G(x, z)}{\partial x^{2}}
$$

here

$$
V(x, z)=\Delta \varphi_{x}(x, z)
$$

with a Dirichlet-type boundary condition

$$
V_{x}(x, \pm 0)=V_{0}(x)=-\frac{K}{2 \beta^{2}} \Delta C_{p \pm}(x)
$$

and a constraint at infinity

$$
\Delta \varphi_{x}(x, z) \rightarrow 0 \quad ; \quad\left(x^{2}+\frac{z^{2}}{\beta^{2}} \rightarrow \infty\right)
$$

Now we use the transformation

$$
T(\chi)=x+i z=\frac{1}{4}\left(\chi+\frac{1}{\chi}\right)+\frac{1}{2}=\frac{1}{4}\left(r e^{i \omega}+\frac{e^{-i \omega}}{r}\right)+\frac{1}{2}
$$

where $\chi=r e^{i \omega}$ is the complex variable in the transformed plane. The entire plane in the physical plane is mapped onto the outside of the unit circle, the chord of the airfoil $(z=0$, $0 \leqq x \leqq 1)$ is corresponds to the unit circle $(r=1,0 \leqq \omega \leqq \pi)$ on the transformed plane. Substituting Eq. (27) into Eq. (23) yields

$$
\nabla^{2} V(r, \omega)=L /\left|T^{\prime}(\chi)\right|^{2}
$$

where

$$
L(r, \omega)=\left(\frac{\partial \omega}{\partial x}\right)^{2} \frac{\partial^{2} G}{\partial \omega^{2}}+2\left(\frac{\partial r}{\partial x} \frac{\partial \omega}{\partial x}\right) \frac{\partial^{2} G}{\partial r \partial \omega}+\left(\frac{\partial r}{\partial x}\right) \frac{\partial^{2} G}{\partial r^{2}}+\frac{\partial^{2} r}{\partial x^{2}} \frac{\partial G}{\partial r}+\frac{\partial^{2} \omega}{\partial x^{2}} \frac{\partial G}{\partial \omega}
$$

The boundary conditions in the transformed plane are

$$
\begin{aligned}
& \left.V(r, \omega)\right|_{r=1}=V_{0}(\omega) \\
& V \rightarrow 0 \quad ; \quad(r \rightarrow \infty)
\end{aligned}
$$

By applying Green's theory to Eq.(28), an integral expression can be obtained as

$$
\begin{aligned}
V\left(r_{1}, \omega_{1}\right) & =\frac{r_{1}^{2}-1}{2 \pi} \int_{0}^{2 \pi} \frac{V_{0}(\omega)}{r_{1}-2 r_{1} \cos \left(\omega-\omega_{1}\right)+1} d \omega \\
& +\frac{1}{2 \pi} \int_{0}^{2 \pi} \int_{1}^{\infty} \log \left[\frac{r_{1}-2 r r_{1} \cos \left(\omega-\omega_{1}\right)+r^{2}}{r_{1}^{2} r-2 r r_{1} \cos \left(\omega-\omega_{1}\right)+1}\right] \frac{L}{\left|T^{\prime}(\chi)\right|^{2}} r d r d \omega
\end{aligned}
$$

If $r_{1} \rightarrow \infty$, the above equation becomes

$$
\int_{0}^{2 \pi} V_{0}(\omega) d \omega=\int_{0}^{2 \pi} \int_{1}^{\infty} \frac{L \log r}{\left|T^{\prime}(x)\right|^{2}} r d r d \omega
$$

In physical plane, Eq. (32) can be written as

$$
\int_{0}^{1}\left[\Delta \varphi_{x}(x,+0)-\Delta \varphi_{x}(x,-0)\right] \omega_{x} d x=\iint_{-\infty}^{\infty} \log r \frac{\partial^{2} G(x, z)}{\partial x^{2}} d x d z
$$


Using an integration by parts and introducing the decay function, we can obtain the final form of the regularity condition

$$
\int_{0}^{1} \Delta u_{a}(x) \omega_{x} d x=\int_{0}^{1}\left[I_{v}(x,+0) G(x,+0)+I_{v}(x,-0) G(x,-0)\right] d x
$$

here

$$
I_{v}(x, \pm 0)=\int_{0}^{\infty} \frac{r r_{x x}-r_{x}^{2}}{r} \exp \left[-2 R_{ \pm}(x) z\right] d z
$$

The above discussion indicates that the prescribed pressure distribution should contain an adjustable parameter to guarantee that the regularity condition is satisfied. Thus the surface pressure distribution is to be prescribed in the form

$$
C_{p s \pm}(x)=F_{ \pm}(\sigma, x)
$$

where $\sigma$ is a parameter that is found as part of the solution. For convenience, the following form of $\mathrm{C}_{p s}$ is used in this paper:

$$
C_{p s \pm}(x)=\sigma F_{ \pm}(x)
$$

In order to assure that the resulting airfoil has a specified trailing edge gap, the following closure condition must be satisfied:

$$
\int_{0}^{1} \Delta w_{s}(x) d x=0
$$

This closure condition can assure that the trailing edge thickness of the current airfoil is always kept equal to that of the initial airfoil.

Although the regularity condition in closed form (34) is obtained from the simple transonic small disturbance theory with Norstrud assumption, its practical utility will be shown by numerical examples in the following section.

\section{NUMERICAL AND COMPUTATIONAL ASPECTS}

In order to discrete the integrals appearing in Eqs. (16) and (17), the range of integration with respect to $\zeta$ is divided into subintervals. Assuming that $R_{ \pm}(\zeta) \zeta$ is constant on each subinterval, Eqs. (16) and (17) can be expressed as

$$
\begin{aligned}
& I_{s}(x, \xi, \pm 0)=\sum_{n=0}^{N} \exp \left[-2 R_{ \pm}(\xi) \zeta_{n}\right] \int_{\zeta_{n}-\frac{1}{2} \Delta \zeta_{n}}^{\zeta_{n}+\frac{1}{2} \Delta \zeta_{n}} \Psi_{\xi x}(x, 0 ; \xi, \zeta) d \zeta \\
& I_{a}(x, \xi, \pm 0)=\sum_{n=0}^{N} \exp \left[-2 R_{ \pm}(\xi) \zeta_{n}\right] \int_{\zeta_{n}-\frac{1}{2} \Delta \zeta_{n}}^{\zeta_{n}+\frac{1}{2} \Delta \zeta_{n}} \Psi_{\xi z}(x, 0 ; \xi, \zeta) d \zeta
\end{aligned}
$$

The range of integration with respect to $\mathrm{x}$ is also divided into subintervals, and on each of subinterval $\Delta u_{s}(x), \Delta u_{a}(x), G(x, \pm 0)$, and $\Delta w_{a}(x)$ are assumed to be con. stants, while $\Delta w_{s}(x)$ is assumed to vary linearly, the final expressions of Eqs. (8) and (9) in discretized form are as follows:

$$
\Delta u_{s}\left(x_{i}\right)=\sum_{k=1}^{t+1} \mu_{i k}^{s} \Delta w_{s}\left(x_{k-\frac{1}{2}}\right)+G_{s}\left(x_{i}\right)-\sum_{k=1}^{I}\left[v_{i k}^{s} G\left(x_{k},+0\right)+\hat{v}_{i k}^{s} G\left(x_{k},-0\right)\right]
$$


$\Gamma$

$$
\Delta w_{a}\left(x_{i}\right)=\sum_{k=1}^{1} \mu_{i k}^{a} \Delta u_{a}\left(x_{k}\right)-\sum_{k=1}^{1}\left[v_{i k}^{a} G\left(x_{k},+0\right)-\hat{v}_{i k}^{a} G\left(x_{k},-0\right)\right]
$$

The discretized form of Eq. (34) and (38) can be expressed as

$$
\begin{gathered}
\sum_{i=1}^{1} \Delta u_{a}\left(x_{i}\right)\left(\omega_{i+\frac{1}{2}}-\omega_{i-\frac{1}{2}}\right)=\sum_{i=1}^{\prime}\left[v_{i}^{\prime} G\left(x_{i},+0\right)+\hat{v}_{i}^{\prime} G\left(x_{i},-0\right)\right] \\
\sum_{i=1}^{\prime}\left[\Delta w_{s}\left(x_{i-\frac{1}{2}}\right)+\Delta w_{s}\left(x_{i+\frac{1}{2}}\right)\right]\left(x_{i+\frac{1}{2}}-x_{i-\frac{1}{2}}\right)=0
\end{gathered}
$$

where the coefficients

$$
\mu_{i k}^{s}, \mu_{i k}^{a}, v_{i k}^{s} . \hat{v}_{i k}^{s}, v_{i k}^{a}, \hat{v}_{i k}^{a}, v_{i}^{\prime}, \hat{v}_{i}^{r}
$$

are the integral expressions on each of the subinterval. The full expressions are omitted here.

In order to improve the convergence, the following modifications have been taken in this paper:

1). A Riegels type of leading edge correction is taken in the method. The purpose of such correction is to remove the singularity at the leading edge of the round-nosed airfoil.

2). In order to increase the ability to deal with the shock, an artificial viscosity term is added to the integral equation method.

3). A Smoothing-relaxation procedure is proposed and used in this paper.

The inverse problem can be solved by the iteration process as follows:

$1)$. The flowfield is solved for an initial airfoil $f_{ \pm}(x)$ by a direct analysis code. From the calculated pressure distribution $C_{p \pm}(x)$, and the target pressure distribution $\mathrm{C}_{\mathrm{ps} \pm}(\mathrm{x})$, the residual $\Delta \mathrm{C}_{\mathrm{p} \pm}(\mathrm{x})=\sigma \mathrm{C}_{\mathrm{ps} \pm}(\mathrm{x})-\mathrm{C}_{\mathrm{p} \pm}(\mathrm{x})$ can be obtained.

2). The adjustable parameter $\sigma$ is determined from the regularity condition (43).

3). The geometric correction $\Delta f_{ \pm}(x)$ is determined by solving the equations (41)-(42). Thus a new geometry is obtained from the following Smoothing-relaxation procedure:

$$
\mathrm{f}_{ \pm}^{\mathrm{n}+1}\left(x_{i}\right)=\mathrm{f}_{ \pm}^{\mathrm{a}}\left(x_{i}\right)+\delta\left[\Delta \mathrm{f}_{ \pm}^{\mathrm{n}+1}\left(x_{i}\right)+0.5\left(\Delta \mathrm{f}_{ \pm}^{\mathrm{n}+1}\left(x_{i-1}\right)+\Delta \mathrm{f}_{ \pm}^{\mathrm{n}+1}\left(x_{i+1}\right)\right)\right]
$$

where $\delta$ is a relaxation factor.

The same process is repeated until the calculated pressure distribution agrees with the prescribed one.

Several test cases are presented to show the validity and applicability of the procedure. A nonisentropic potential solver for $2-D$ transonic flow ${ }^{14}$ is used as the analysis code.

In the first example, the target pressure distribution is taken from the result of RAE2822 airfoil, the free stream Mach number is 0.73 , and the angle of attack is $\alpha=2.05^{\circ}$. The initial airfoil is NACA0012 airfoil, the initial angle of attack is $\alpha=0.0$. After 12 design cycles, the RAE2822 airfoil is already recovered. Indeed the designed airfoil is rotated in a clockwise direction by $2.05^{\circ}$ with respect to the original airfoil. This is because the initial angle of attack is chosen to be $0^{\circ}$, the angle between the free stream and the $\mathrm{X}-\mathrm{Axi}$ is always $0^{\circ}$ during iterations. Fig. 1 shows the target and initial 
pressure distribution. Fig. 2 shows the convergence procedure of the pressure distribution.

The second example is chosen to show the function of the regularity condition. In Ref. [15], Strand gave a velocity distribution which did not satisfy the Lighthill's three integral constraints in incompressible flow, hence there is no airfoil corresponding to this velocity distribution. Strand modified this velocity distribution according to the Lighthill's constraints, and designed the airfoil corresponding to the modified velocity distribution, the results is showed in Fig. 3. Now, we calculate this example using the present method. For convenience, only the velocity distribution on the lower surface is multiplied by the free parameter $\sigma$. After 7 design cycles, the converged solution is obtained, the velocity distributions are given in Fig. 4. In this case, the output velocity distribution on the lower surface is not consistent with input velocity distribution, this is because the free parameter $\sigma$ is not equal to 1 , but equal to 1.07 . The results show that the method can adjust the improperly input velocity distribution to the acceptable velocity distribution automatically, and design the airfoil corresponding to the modified velocity distribution. Comparing the figures the present result is closer with input data than Strand's one. On the other hand, if the regularity condition is not included in the method, the design procedure will not converge for this example.

\section{CONCLUSIONS}

A regularity condition in closed form for transonic flow is presented in this paper, and a well posed inverse design method for transonic airfoil is formulated. The results show that the method is a reliable and efficient method for the design of airfoil at transonic speeds. When the target pressure distribution is not properly given, the code can adjust the target pressure distribution automatically and design the airfoil corresponding to the modified pressure distribution.

\section{REFERENCES}

1. Slooff, J. W., “A survey of computational Methods for Subsonic and Transonic Aerodynamic Design", Paper at ICIDES- I, Austin, Tex., U.S.A., 1984.

2. Volpe, G. and Melnik, R.E., "The Role of Constraints in the Inverse Design Problem for Transonic Airfoils", AIAA Paper 81-1233, 1981.

3. Carlson, L. A., “Transonic Airfoil Design Using Cartesian Coordinates," NASA CR-2578, 1976.

4. Tranen, T. L., " A Rapid Computer Aided Transonic Airfoil Design Method" , AIAA Paper 74-501, 1974. 
5. Henne, P. A., “An Inverse Transonic Wing Design Method”, AIAA Paper 80-0330, 1980.

6. Shankar, V., "A Full Potential Inverse Method Based on a Density Lineaerization Scheme for Wing Design", AIAA Paper 81-1234. 1981.

7. Davis Jr., W. H., "Technique for Developing Design Tools from the Analysis Methods of Computational Aerodynamics", AIAA Paper 79-1259, 1979.

8. McFadden, G. B., "An Artificial Viscosity Method for the Design of Supercritical Airfoils”, Ph. D. Thesis, N. Y. University, 1979.

9. Fray, J. M. J., Slooff, J. W. et al., "Inverse Method with Geometric Constraints for Transonic Airfoil Design”, Int. J. for Numerical Methods in Engineering, Vol. 22, pp. 327-339, 1986.

10. Greff, E., Mantel, J., "An Engineering Approach to the Inverse Transonic Wing Design Problem", Comm. Appl. Num. Meth., Vol. 2, pp. 47-56,1986.

11. Takanashi, S., "Iterative Three-Dimensional Transonic Wing Design Using Integral Equations", J. Aircraft, NO.8 Vol.22, 1985.

12. Lighthill, M. J., "A New Method of Two-Dimensional Aerodynamic Design", ARC RM 2112, April. 1945.

13. Nфrstrud, H., "High Speed Flow Past Wings", NASA CR-2246, 1973.

14. Zhu Z. Q., Bai Xue-song, "The Computation of Transonic Analysis and Design", Acta Mechanica, 78, pp 81-94, Springer Verlag, 1989.

15. Strand, T., "Exact Method of Designing Airfoils with Given Velocity Distribution in Incompressible Flow”, J. Aircraft, Vol. 10, No. 11, 1973. 
Third Intemational Conference on Inverse Design Concepts and Optimization in Engineering Scicnces (ICIDES-III). Editer: G.S. Dulikravich. Washington D.C. Oclober 23-25. 1991.
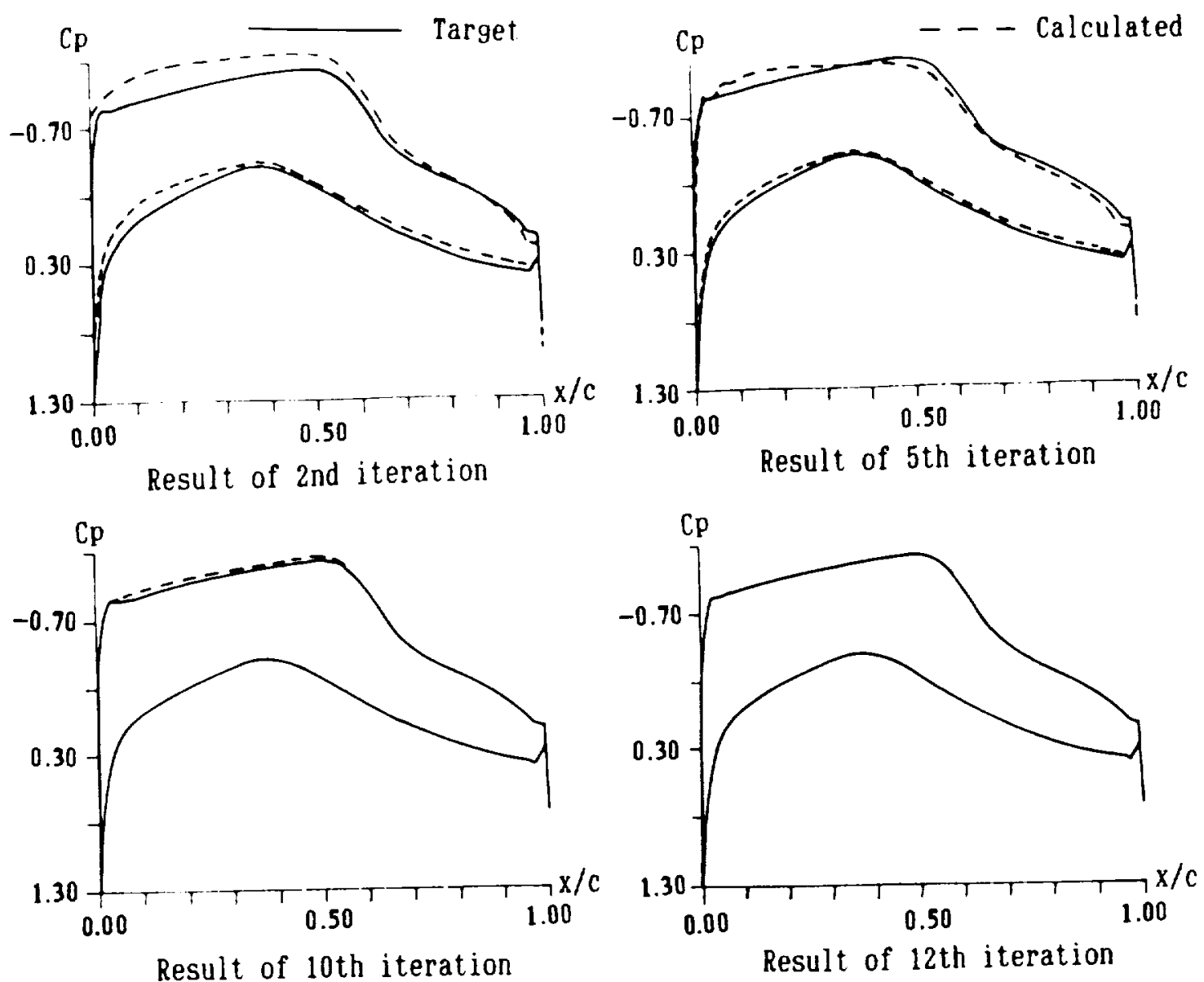

Fig. 2 Convergence history of the pressure distribution (exanple 1)

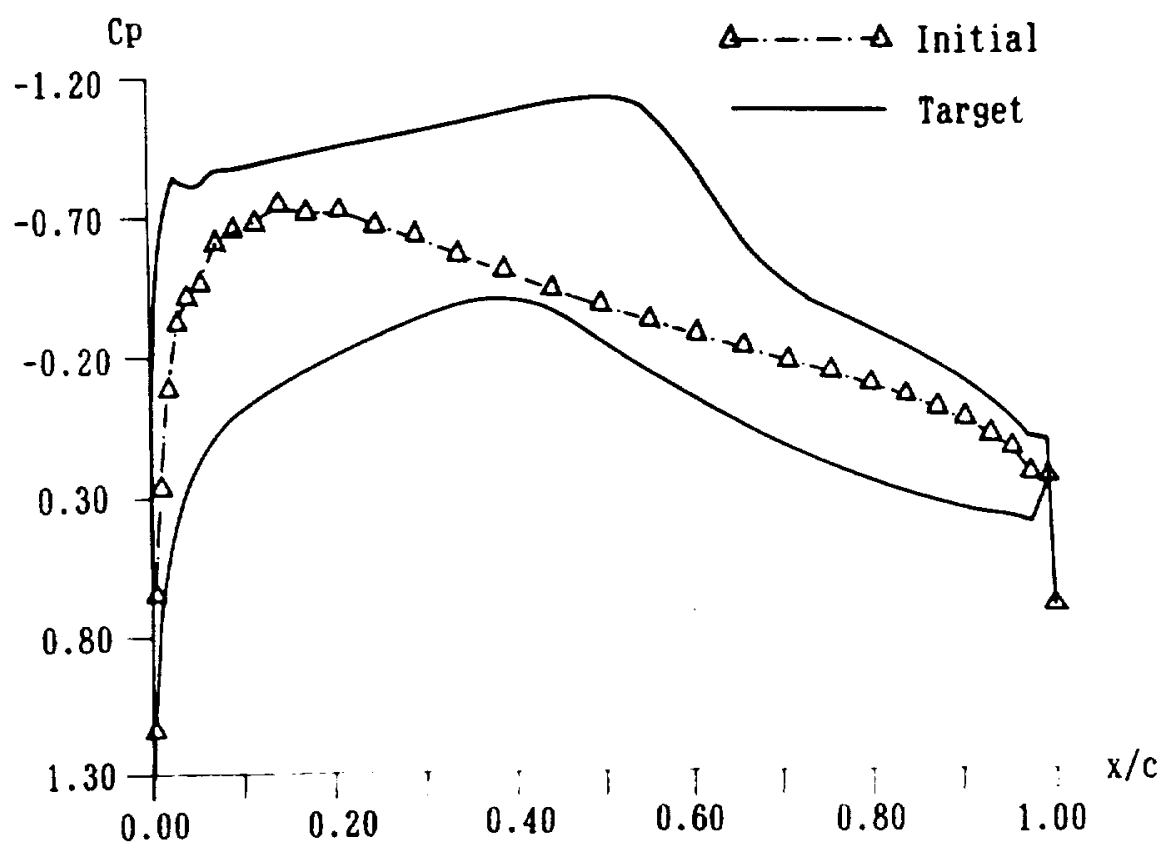

Fig. 1 Comparison of initial, target pressure distribution (example 1) 


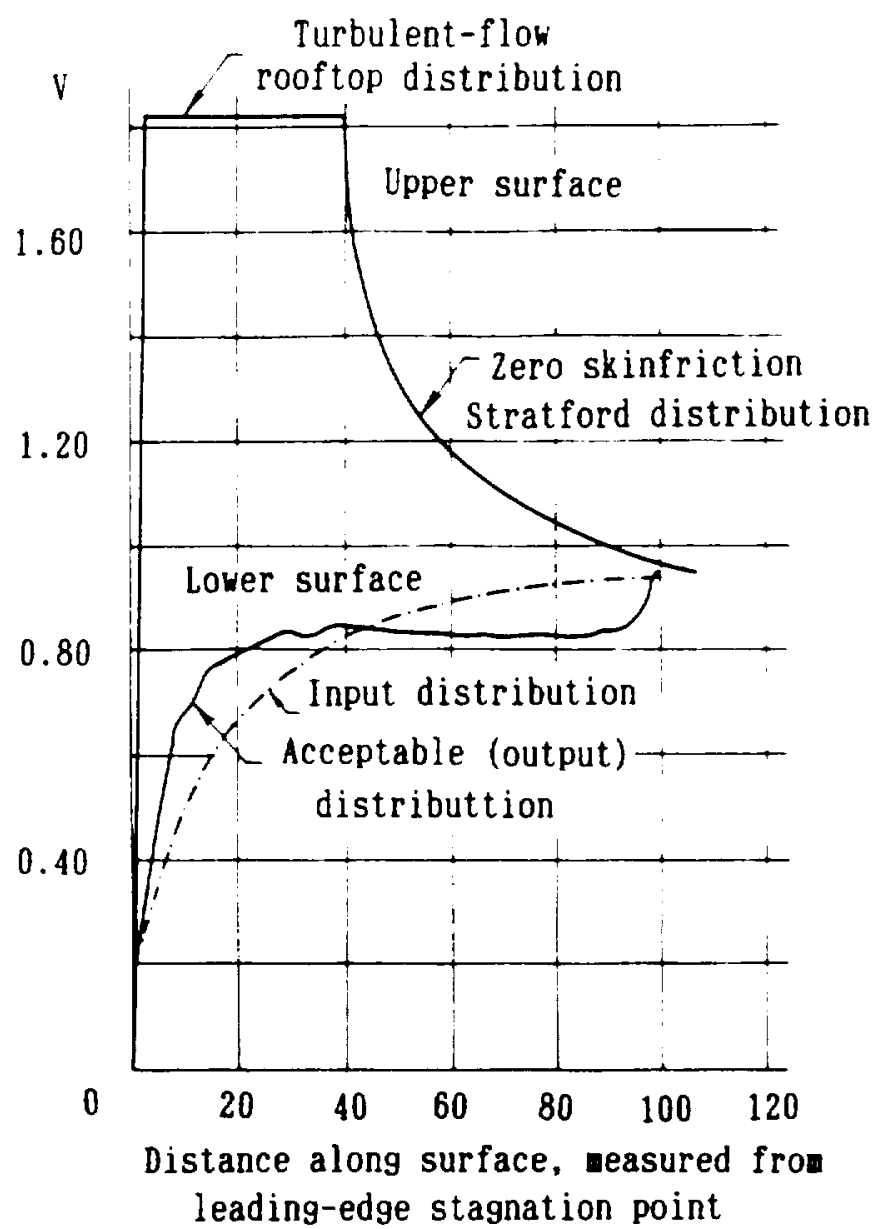

Fig. 3 The input and acceptable velocity distribution given by Strand 


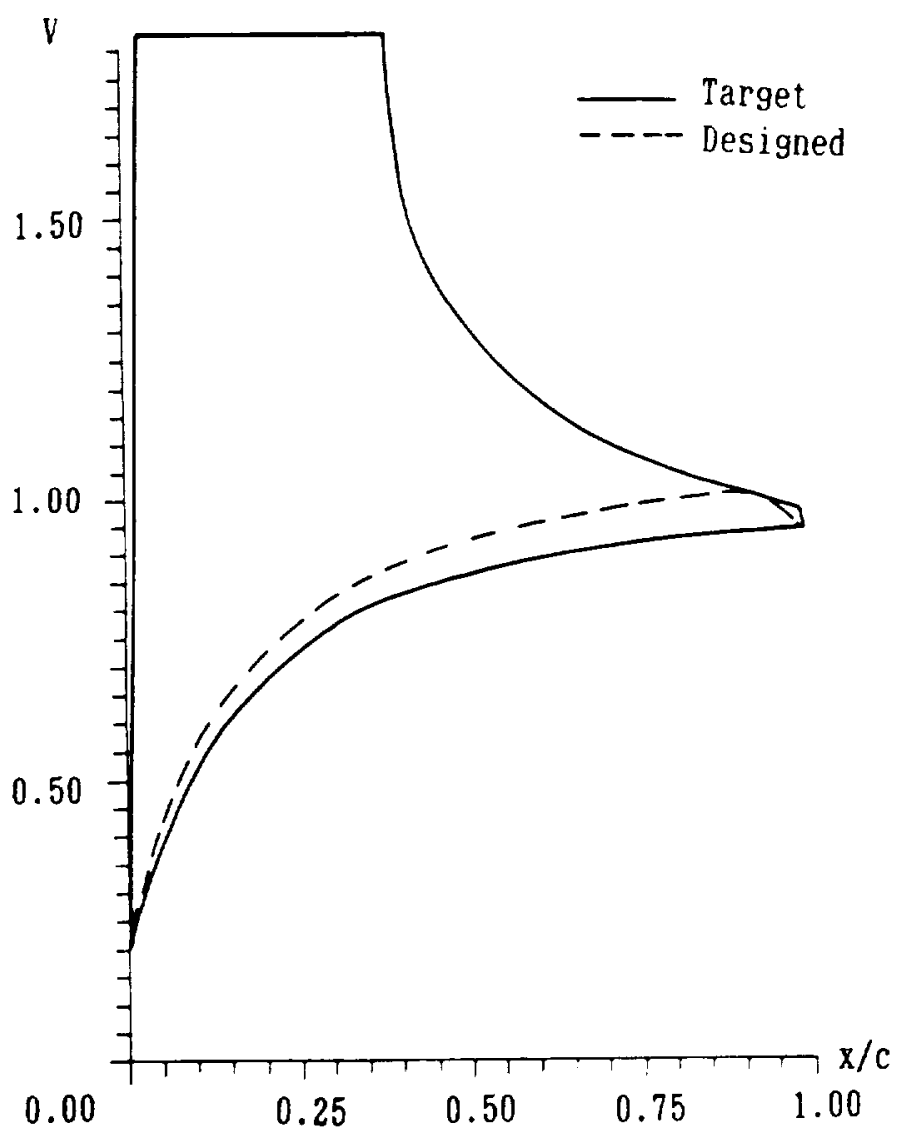

Fig. 4 The input and designed velocity distribution (example 2) 